\title{
CNS Modulation of Pancreatic Endocrine Function
}

\author{
Multiple Modes of Expression \\ D. A. Bereiter, F. Rohner-Jeanrenaud, H.-R. Berthoud, and B. Jeanrenaud \\ Laboratoires de Recherches Métaboliques, Département de Médecine, Université de Genève, Genève, Switzerland
}

Summary. The involvement of the CNS in pancreatic hormone release has been studied. 1.) It has been shown that one source of vagal efferent fibers capable of facilitating insulin secretion originated in the rostral half of the nucleus ambiguus. 2.) Acute lesions of the ventromedial hypothalamus resulted in hyperinsulinaemia that could be abolished by acute vagotomy. 3.) Chronic lesions of the ventromedial hypothalamus increased secretion of insulin and glucagon, and decreased secretion of somatostatin when the pancreas was subsequently isolated and perfused. These changes were attributed to altered cholinergic activity related to previous ventromedial hypothalamic lesions as they could be reversed toward normal by atropine infusion or mimicked by the cholinergic agonist, methacholine. 4.) Electrical stimulation of the lateral hypothalamus in anaesthetized rats produced both an inhibitory component of insulin secretion, probably related to adrenergic stimulation, and a stimulatory component, probably due to the release into the blood of factor(s) that promote insulin secretion. 5.) The anatomical organization of brain of the genetically obese $(o b / o b)$ mice is abnormal. These abnormalities could be involved in the endocrinological disturbances of these animals.

Key words: Acute ventromedial hypothalamic lesions (VMH), chronic VMH lesions, lateral hypothalamus stimulation, nucleus ambiguus stimulation, insulin secretion, glucagon secretion, somatostatin secretion, brain organization of obese $(o b / o b)$ mouse

Humoral factors such as metabolic substrates, together with systemically or locally released hormones, are major secretagogues for the control of insulin and glucagon secretion from the endocrine pancreas. However, considerable evidence suggests a neural contribution to the control of the endocrine pancreas as well. The autonomic nervous system via the vagus and splanchnic nerves probably provides the anatomical basis for the efferent innervation of the pancreas [33]. Indeed, a vagal facilitatory [5] and a splanchnic inhibitory [27] modulation of insulin release has been demonstrated with the in situ perfused pancreas preparation, a preparation that allows direct neural stimulation to be studied under controlled perfusion (substrate) conditions. Both the vagal and splanchnic innervation of the pancreas have been shown to contribute to hormone secretion under physiological conditions known to depend on neural sensory afferent information. Thus, the early rise in insulin levels after oral ingestion appears to be gustatoryevoked via oropharyngeal sensory afferent nerves [7] and is absent in animals previously vagotomized subdiaphragmatically [26]. A rapid rise in glucagon and a suppression of insulin release accompany a fall in systemic blood pressure that is dependent on sinus nerve baroreceptor afferents and splanchnic nerve connections to the pancreas [21]. Furthermore, splanchnic nerve transection elevates basal insulin levels more markedly under hypoglycaemic than under normoglycaemic conditions [27].

The above examples serve to illustrate that both major branches of the autonomic nervous system contribute to the control of pancreatic hormone release; however, the sites for integration and modulation of such autonomic efferent outputs within the central nervous system (CNS) are not well understood. The following paper summarizes much of the work done in our laboratory directed towards understanding the involvement of the CNS in pancreatic hormone release.

\section{Caudal Brain Stem Localization of Pancreatic Efferents}

Stimulation of the peripheral vagus nerve leads to an elevation in circulating insulin levels $[5,11,22]$. The origin of these vagal efferent fibers within the caudal 

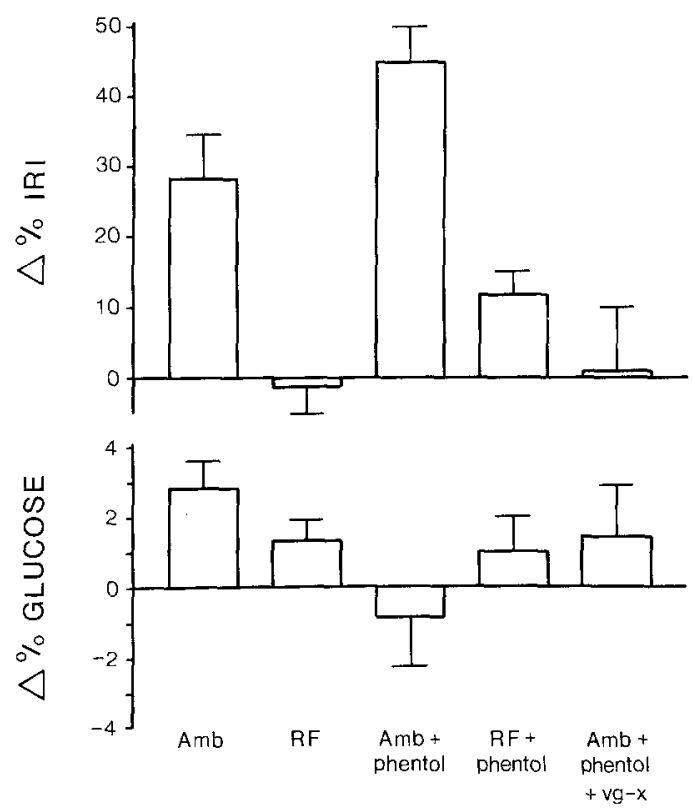

Fig. 1. The effect of unilateral brain stem electrical stimulation on plasma insulin (IRI) and glucose (BG) levels. The response to the first minute of a 3-minute stimulation period is shown, expressed as percentage change from baseline values (Mean \pm SEM). Experimental groups: $\mathrm{Amb}=$ nucleus ambiguus, $\mathbf{n}=27 ; \mathrm{Amb}+$ phentol $=\mathrm{Amb}+$ phentolamine $(5 \mathrm{mg} / \mathrm{kg}), \mathrm{n}=10 ; \mathrm{Amb}+$ phentol $+\mathrm{Vg}-\mathrm{X}=\mathrm{Amb}+$ phentolamine $(5 \mathrm{mg} / \mathrm{kg})+$ bilateral cervical vagotomy, $\mathrm{n}=4 ; \mathrm{RF}=$ medullary reticular formation, $\mathrm{n}=18 ; \mathrm{RF}+$ phentol $=\mathrm{RF}+$ phentolamine $(5 \mathrm{mg} / \mathrm{kg}), \mathrm{n}=9$. Stimulus parameters $=50 \mu \mathrm{A}, 30 \mathrm{cps}, 0.2 \mathrm{~ms}$

brain stem are not known with certainty, but two recent anatomical reports strongly suggest that both the nucleus ambiguus (Amb) as well as the dorsal motor nucleus of the vagus nerve (DMX) contribute to the vagal innervation of the pancreas. Using the retrograde axonal transport marker, horseradish peroxidase (HRP), Laughton and Powley [23] have shown that direct injection of HRP into the pancreas labels neurones in both Amb and DMX bilaterally in the brain stem. Directly applying HRP to the subdiaphragmatic vagus nerve [10] yields more extensive labelling in Amb and DMX than pancreatic injection, but essentially confines the labelled neurones to Amb and DMX.

To substantiate these anatomical findings, we have begun a series of experiments designed to investigate the hormonal response to electrical stimulation of discrete brain stem regions in male rats anesthetized with an $\alpha$-chloralose/urethane mixture. Jugular vein blood samples were taken to monitor the change in plasma glucose (BG) and immunoreactive insulin (IRI) before, during and after unilateral electrical brain stem stimulation $(50 \mu \mathrm{A}, 30 \mathrm{~Hz}$, $0.2 \mathrm{~ms}$ duration, for $3 \mathrm{~min}$ ). Figure 1 summarizes the results after the first minute of stimulation. All data for blood glucose and immunoreactive insulin levels are presented as percent change from baseline. As shown, by $1 \mathrm{~min}$ following unilateral rostral Amb stimulation, there is an approximately $30 \%$ rise in plasma insulin levels which is accompanied by a slight $(2.8 \%)$ increase in blood glucose levels. All stimulation points later found to be within $500 \mu \mathrm{m}$ of Amb are included in this group. This rise in Amb-stimulated insulin levels is to be compared to those points dorsal and medial to Amb (labelled as RF in Figure 1) which show no change $(-1.2 \%)$ in insulin or blood glucose $(1.3 \%)$ levels.

The increased insulin release during Amb stimulation persisted for the duration of the 3 min stimulation period; however by the second or third minute, several individual animals additionally had a rise in blood glucose levels that may have contributed to the increased insulin levels. Animals receiving brain stem stimulation in the reticular formation never demonstrated a rise in insulin levels.

To evaluate the observation of the Amb-induced rise in insulin, independent of concomitant increases in blood glucose levels, the $\alpha$-blocker, phentolamine $(5 \mathrm{mg} / \mathrm{kg}$, IV) was given 30 minutes prior to brain stem stimulation. As shown in Figure 1, Amb stimulation in the presence of phentolamine evoked a larger insulin release for each of the 3 min of stimulation with no change in blood glucose levels compared to the response seen without phentolamine. This Amb-induced elevation of insulin levels could be blocked by bilateral cervical vagotomy ( $\mathrm{Vg}-\mathrm{X}$ in Fig. 1). Phentolamine also promoted a slight rise in insulin levels during reticular formation stimulation. The exaggerated Amb-induced insulin response in the presence of phentolamine without a change in blood glucose levels contrasts with the data of Frohman et al. [11], where vagal stimulation in the presence of phentolamine was not exaggerated compared to vagal stimulation alone, nor was the slight rise in glycemia blocked by phentolamine. This could be due to a species variation (dogs versus rats). However, the difference might also be explained by the source of the stimulation (peripheral nerve versus CNS sites). The ventrolateral brain stem (near the $A m b$ ) is also a major pathway for the $A_{1}$ and $A_{5}$ catecholamine containing cell groups [32], whose fibers contribute to the spinal sympathetic system. Stimulation in this region in the absence of phentolamine may have evoked a concurrent sympathetic as well as parasympathetic (vagal motoneurons) output. In the presence of phentolamine, this concurrent sympathetic outflow would be diminished, either by the action of the drug directly within the brain stem or by a release from $\alpha$-adrenergic inhibition at the 
pancreas (B-cell) level as has been shown previously [19]. Peripheral vagus nerve stimulation, on the other hand, would not be expected to alter directly sympathetic tone at the pancreas level.

The data demonstrate, on physiological grounds, that one source of vagal efferent fibers capable of facilitating insulin release originates in the rostral half of the nucleus ambiguus, corroborating the anatomical findings of Laughton and Powley [23]. We have not yet systematically explored the dorsal medial region of the caudal brain stem to ascertain the effectiveness of DMX stimulation on insulin release. However, it should be mentioned that several stimulation sites facilitatory for insulin release (data not shown) were found in the lateral portion of the nucleus of the solitary tract (NTS). This nucleus receives the majority of chorda tympani, glossopharyngeal and vagal afferent fibers [2] and distributes fibers to both the DMX and Amb [24]. Stimulation of the NTS might be expected to facilitate insulin release by: a) driving vagal motoneurons directly, or b) mimicking afferent sensory input. However, the involvement of the NTS and DMX in the control of insulin release is not known at present and merits further investigation.

\section{Acute Ventromedial Hypothalamic (VMH) Lesions}

Since the early lesion studies by Anand and Brobeck [1], the concept of CNS control of metabolic function has been dominated by the apparent role of the ventral hypothalamus $[9,28]$. Indeed, the classical chronic VMH lesion syndrome is accompanied by severe alterations in metabolic homeostasis, including hyperinsulinaemia, hyperphagia and increased fat accretion, but the exact nature of VMH modulation of pancreatic function is not well understood. This is due in part to the use of the chronic VMH lesion experimental paradigm.

To obviate the problems of interpretation of chronic VMH lesions on pancreatic hormone release [8], acute bilateral VMH lesions were made in anaesthetized (pentobarbital) male rats. As shown in Figure 2, acute VMH lesions (20-25 mCoul) caused an increase in plasma insulin levels that was significantly higher than that of cortex-lesioned controls by $30 \mathrm{~min}$ after lesioning. All experiments were done under a constant glucose infusion ( 7 to $8 \mathrm{mg} / \mathrm{kg}$ / min). Such lesions had no significant effect on blood glucose levels. The exaggerated basal release of insulin in VMH-lesioned animals could be blocked by bilateral subdiaphragmatic vagotomy, the transection being made approximately $50 \mathrm{~min}$ after lesioning (see Fig. 2). In the absence of the constant glucose

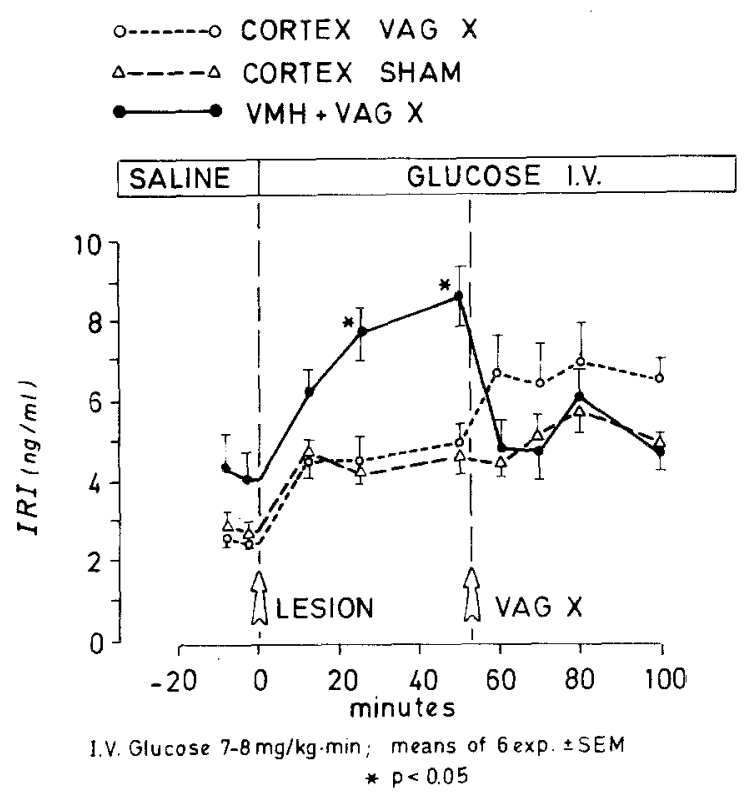

Fig. 2. Effect of acute bilateral VMH lesions and bilateral subdiaphragmatic vagotomy $(\operatorname{VagX})$ on plasma insulin (IRI) levels. $(\bullet)=$ $\mathrm{VMH}$ lesion plus VagX, $\mathrm{n}=6 ;(\mathrm{O}--\mathrm{O})=$ Cortex control lesions plus $\operatorname{VagX}, \mathrm{n}=6 ;(\triangle-\Delta)=$ Cortex control lesions plus sham VagX, $n=6$. All experiments were performed during a constant glucose infusion, 6 to $7 \mathrm{mg} / \mathrm{kg} / \mathrm{min}$. $* \mathrm{P}<0.05$, two-tailed $\mathrm{t}$-test

infusion, VMH-lesioned animals also showed elevated insulin levels relative to cortex controls, but the magnitude and latency to peak values were attenuated.

These data demonstrate that acute VMH lesions cause an increase in circulating insulin levels within $30 \mathrm{~min}$. The effect certainly cannot be attributed to such secondary effects as hyperphagia or pancreatic islet hypertrophy as occurs in the chronic VMH animal. Successful blockade of the exaggerated insulin release by vagotomy confirms recent investigations showing that the chronic VMH syndrome (with hyperinsulinaemia) is not seen in vagotomized animals [17] nor in islet-transplanted animals previously given streptozotocin [18]. Taken together, these results strongly suggest that the effect of $\mathrm{VMH}$ lesions on pancreatic hormone release is, at least in part, neural and probably requires an intact vagal innervation of the pancreas. However, a more indirect vagal effect via the release of gut hormones is not necessarily excluded.

The underlying neural substrate within the CNS responsible for the $\mathrm{VMH}$ lesion effect on insulin release is not known; however, recent neuroanatomical data provide some insight to this problem.

First, it must be stated that effective VMH lesions generally destroy much more than just cell bodies lying within the ventromedial hypothalamic nucleus. 

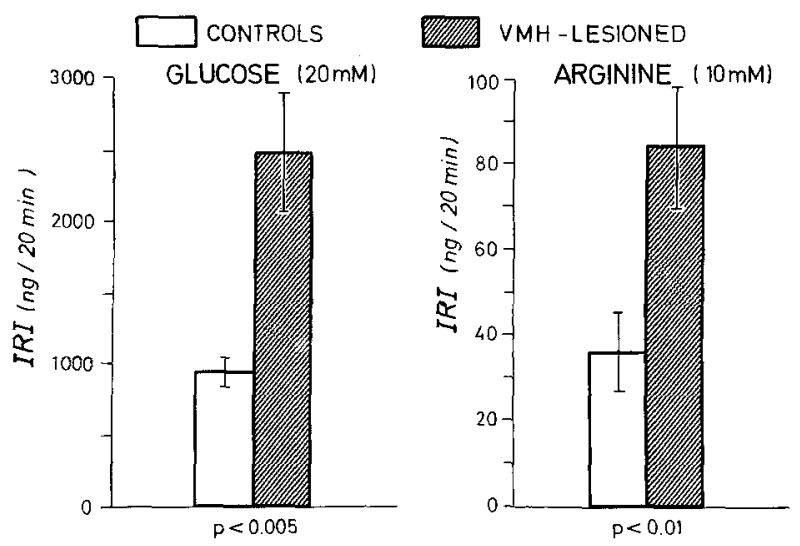

Fig. 3. Immunoreactive insulin (IRI) release by perfused pancreas from control and VMH-lesioned rats. Perfusion medium was a Krebs-Ringer bicarbonate buffer with human serum albumin $(2.5 \mathrm{~g} / \mathrm{l})$ plus iniprol (2000 anti-protease units/ml). The stimulus for insulin release was glucose at $20 \mathrm{mmol} / 1$ (left panel) and arginine $(10 \mathrm{mmol} / 1)$ without glucose (right panel). Stimulation period: $20 \mathrm{~min}$. Each bar is the mean of 5 to 8 perfusions \pm SEM. $P$ values for controls (open bars) vs VMH-lesioned rats (hatched bars) were determined by t-test
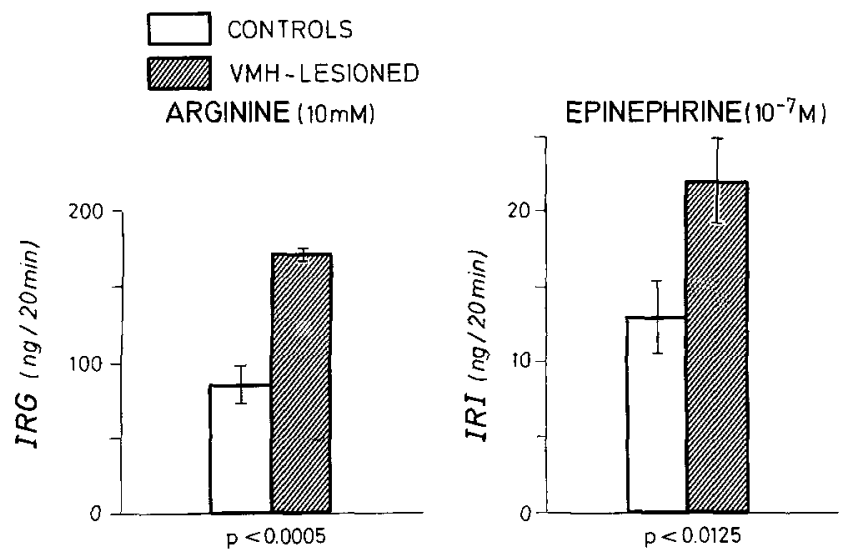

Fig. 4. Immunoreactive glucagon (IRG) release by perfused pancreas from controls and VMH-lesioned rats. Perfusion medium was the same as Fig. 3. Stimuli of glucagon release were arginine $(10 \mathrm{mmol} / 1)$ without glucose (left panel) and epinephrine $\left(10^{-7} \mathrm{~mol} / \mathrm{l}\right)$ plus $2.5 \mathrm{mmol} / 1$ glucose (right panel). Stimulation period: $20 \mathrm{~min}$. Each bar is the mean of 5-8 perfusions \pm SEM. $P$ values for controls (open bars) vs VMH-lesioned rats (hatched bars) were determined by t-test

Of particular concern here is damage to fibres of passage. In fact, lesions confined to the ventromedial nucleus are usually not successful in causing the VMH syndrome [12]. Efferent fibers from the paraventricular hypothalamic nucleus (PVN) may be critical for the regulation of food intake [13] and these fibers sweep ventrally and posteriorly to pass through and lateral to the ventromedial nucleus [31]. Furthermore, the PVN has been shown to make reciprocal connections directly with brain stem vagal nuclei [30]. Although PVN lesions have not been shown to alter vagal motoneurone activity or insulin release directly these anatomical data provide a framework to understand how discrete hypothalamic lesions could affect the neural control of pancreatic endocrine function as expressed by vagal motoneurone tone.

\section{Chronic VMH Lesions}

As mentioned previously, VMH lesions have widespread autonomic, endocrine and metabolic effects that are particularly difficult to interpret in the chronic VMH animal in vivo [8]. However, the in vitro isolated perfused pancreas preparation provides a method to study the secretory activity of the endocrine pancreas in the absence of direct neural and/or unwanted humoral factors in chronic VMH-lesioned animals.

The following series of experiments were designed to test: a) whether the isolated perfused pancreas of VMH animals secretes more insulin than controls in the absence of direct neural input; b) whether other hormones such as glucagon or somatostatin also demonstrate hypersecretion to secretagogues in the VMH animal; and c) the pharmacological response to cholinergic agonists and antagonists, based largely on the assumption that the VMH syndrome requires an intact parasympathetic autonomic nerve supply.

The data shown below are derived from VMHlesioned (and control) female rats. The lesioned animals were fed ad lib for 3 days before being pair-fed to controls for an additional 4 days. This regimen allowed the effectiveness of the VMH lesion to be assessed, but prevented excess obesity at the time of the removal of the pancreas for perfusion [29]. As shown in Figure 3, the magnitude of the insulin response to a glucose $(20 \mathrm{mmol} / \mathrm{l})$ or arginine $(10 \mathrm{mmol} / \mathrm{l})$ challenge was considerably greater in pancreases from VMH-lesioned animals compared to controls. The biphasic pattern of insulin release was however, qualitatively unchanged (data not shown). The glucagon response to arginine $(10 \mathrm{mmol} / \mathrm{l})$ or epinephrine $\left(10^{-7} \mathrm{~mol} / \mathrm{l}\right)$ was also quantitatively greater in VMH animals compared to controls (Figure 4), but again, was not qualitatively different as both groups showed equivalent biphasic release patterns to secretagogues.

The chronic VMH syndrome requires an intact pancreatic vagal nerve supply [18]. Since the insulin response to vagal nerve stimulation [5] and to pancreas perfusion with acetylcholine [20] is mediated by 
Table 1. Effects of methacholine and atropine on insulin, glucagon and somatostatin secretion from perfused pancreases of control and VMH-lesioned rats

\begin{tabular}{lrrr}
\hline Conditions $^{\mathrm{a}}$ & IRI & IRG & IRS \\
\hline A. Control + methacholine & $100 \% \mathrm{~b}$ & 100 & 100 \\
B. Control + methacholine + atropine & 21 & 22 & 92 \\
C. VMH-lesioned + methacholine & 476 & 167 & 62 \\
D. VMH-lesioned + methacholine & 36 & 29 & 100 \\
$\quad+$ atropine & & & \\
\hline
\end{tabular}

a All perfusions were made in the presence of glucose $(2.5$ $\mathrm{mmol} / \mathrm{l})$ and methacholine $(100 \mu \mathrm{mol} / \mathrm{l})$. Atropine concentration was $25 \mu \mathrm{mol} / 1$

b All data are expressed as the percentage of the control (A) response (ng released/20 $\mathrm{min}$ ). Each value represents the mean of 7 to 9 perfusions

Data in press (Diabetologia)

muscarinic cholinergic receptors, we sought to determine if perfused pancreas from VMH-lesioned animals showed any quantitative hormonal response differences to muscarinic agonists (methacholine) or antagonists (atropine) compared to controls. All perfusions were made under constant glucose (2.5 $\mathrm{mmol} / \mathrm{l})$ conditions, plus methacholine $(100 \mu \mathrm{mol} / \mathrm{l})$, with or without atropine $(25 \mu \mathrm{mol} / \mathrm{l})$. The data for insulin (IRI), glucagon (IRG) and somatostatin (IRS) release to these perfusion conditions for VMH-lesioned and control animals are shown in Table 1 and expressed as a percentage of the response to methacholine alone in the control animals. As seen in Table 1, VMH-lesioned animals had an augmented insulin and glucagon response to methacholine that was completely blocked by atropine. Somatostatin release was significantly decreased by methacholine in VMH-lesioned animals compared to controls, a decrease that was normalized by atropine.

These data demonstrate that in vitro, the pancreas from VMH-lesioned animals secretes more insulin and glucagon than that of controls. Furthermore, VMH-lesioned animals respond to the cholinergic agonist methacholine with increased insulin and glucagon secretion and these effects can be blocked by atropine.

One explanation for these results may be increased islet size or number due to VMH lesioning or an increase in the number of receptors at the cellular level capable of responding to the various secretagogues used. Indeed, Han et al. [14] have shown that even tube-feeding VMH-lesioned animals with equivalent intake matched to that of controls leads to an increase in islet area, suggesting that $\mathrm{VMH}$ lesions lead to significant histological changes in the pancreas even in the absence of hyperphagia.
The fact that the chronic VMH syndrome can be blocked by vagotomy suggests that such lesions may alter vagal electrical activity to the pancreas or concurrently, that the vagus nerve exerts some trophic influence on pancreatic function.

\section{Acute Lateral Hypothalamic Area (LHA) Stimulation}

The hypothesis of reciprocal hypothalamic "hunger and satiety" centers for the control of feeding behaviour [28] has often been extrapolated to explain a reciprocal hypothalamic control of pancreatic hormone release. The lateral hypothalamic area (LHA) and the ventromedial hypothalamus (VMH) have been described as generating predominantly parasympathetic and sympathetic responses respectively, with regard to the regulation of insulin release and the control of blood glucose [33]. Although VMH stimulation consistently increases blood glucose levels with a parallel suppression of insulin release, the effects of LHA stimulation have been contradictory and have not clearly substantiated the "reciprocal center" hypothesis [6].

To investigate further the effects of electrical stimulation of the LHA on pancreatic endocrine function, anaesthetized (pentobarbital) male rats received bilateral LHA stimulation $(250 \mu \mathrm{A}, 50 \mathrm{~Hz}$, $0.2 \mathrm{~ms}$ ) in the presence of a continuous low glucose infusion to obtain a constant blood glucose level for all experiments. Jugular vein blood samples were taken at appropriate time intervals for the measurement of insulin release and blood glucose levels. Stimulation of the LHA caused a small increase in insulin levels, but it was accompanied by a significant elevation of blood glucose levels as well (Figure 5, control). Since this suggested a sympathetic-like response, the $\alpha$-adrenergic blocker (phentolamine, $90 \mu \mathrm{g} / \mathrm{kg} / \mathrm{min}$ ) was infused simultaneously with the glucose. As seen in Figure 5, phentolamine blocked the LHA-induced rise in blood glucose levels and revealed a highly significant increase in insulin release. These results may be interpreted to show that LHA stimulation simultaneously evoked parasympathetic as well as sympathetic-like responses in terms of insulin release and blood glucose levels. However, all attempts to block an apparent autonomic nervous system-mediated release of insulin during LHA stimulation in the presence of phentolamine were unsuccessful. As further seen in Figure 5 , neither bilateral subdiaphragmatic vagotomy, atropine $(300 \mu \mathrm{g} / \mathrm{kg} / \mathrm{min})$ nor the $\beta$-adrenergic blocker, propranolol $(60 \mu \mathrm{g} / \mathrm{kg} / \mathrm{min})$ significantly reduced the insulin release seen following LHA stimulation in the presence of phentolamine. 


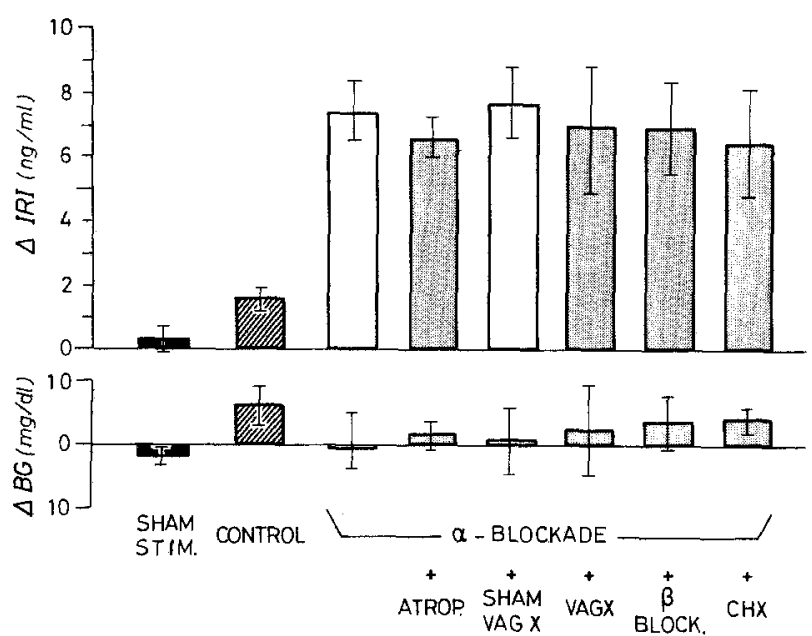

Fig. 5. Effect of bilateral LHA stimulation on peripheral plasma levels of insulin (IRI) and glucose (BG) in pentobarbital anaesthetized rats. All experiments were performed during a constant glucose infusion $(6 \mathrm{mg} / \mathrm{kg} / \mathrm{min})$ and the data represent the changes from baseline observed at $2 \mathrm{~min}$ of a $5 \mathrm{~min}$ stimulation period. Experimental groups: sham stim $=$ electrodes lowered to approx. $1 \mathrm{~mm}$ above LHA, no current, $\mathrm{n}=3$; control = LHA stimulation alone, $\mathrm{n}=7 ;$ LHA stimulation + phentolamine $(90 \mu \mathrm{g} / \mathrm{kg} / \mathrm{min}), \mathrm{n}$ $=7$; LHA stimulation + phentolamine + atropine $(300 \mu \mathrm{g} / \mathrm{kg} /$ $\min ), \mathrm{n}=9$; LHA stimulation + phentolamine + sham vagotomy, $\mathrm{n}=7$; LHA stimulation + phentolamine + bilateral subdiaphragmatic vagotomy (VagX), $\mathrm{n}=9$; LHA stimulation + phentolamine + propranolol $(60 \mu \mathrm{g} / \mathrm{kg} / \mathrm{min}), \mathrm{n}=7 ; \mathrm{CHX}=$ LHA stimulation + phentolamine + spinal cord transection at $\mathrm{T}_{1}$, $\mathrm{n}=4$. Stimulus parameters: $250 \mu \mathrm{A}, 50 \mathrm{cps}, 0.2 \mathrm{~ms}$

Unlike the vagally-mediated effects on insulin release due to ventral brain stem stimulation or acute VMH destruction as reported earlier, this LHAinduced rise in insulin levels does not appear to be mediated by classical autonomic neural output pathways. Instead, we suggest that this rise in insulin levels supports previous concepts of an LHA humoral factor which facilitates insulin release [15, 16]. Alternative explanations include: a) the release of a novel peptide transmitter from autonomic nerve terminals that would be resistant to adrenergic or cholinergic pharmacological blockers, or b) LHAinduced changes in cardiovascular function sufficient to alter systemic insulin levels independent of a direct B-cell neural influence. The exact nature of this LHA-induced hyperinsulinaemia seen during phentolamine treatment must await further investigations.

\section{Neuroanatomy in the Genetically Obese (ob/ob) Mouse}

Since considerable evidence suggests that the CNS significantly affects insulin secretion, then the contrary, namely that chronic disturbances in insulin se-

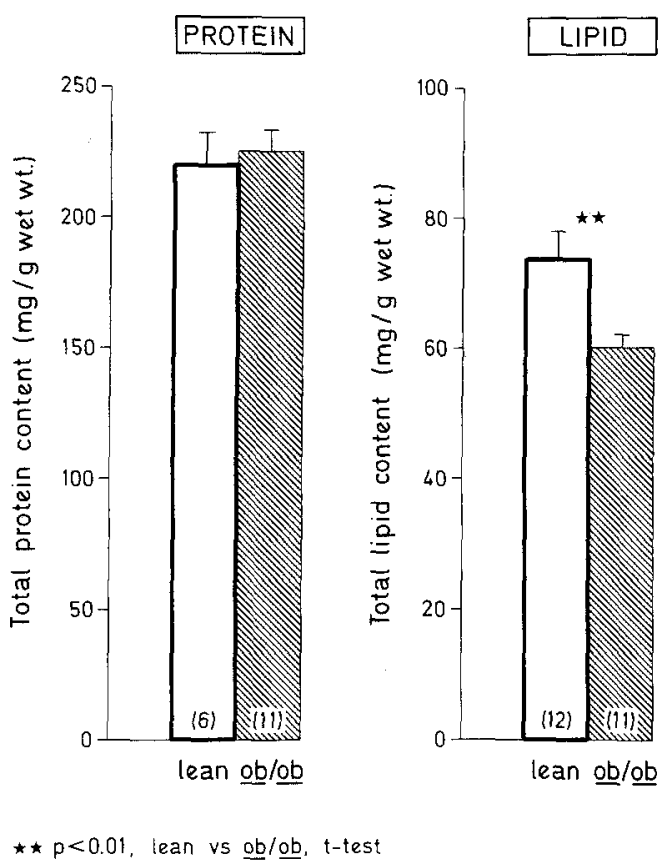

Fig. 6. Total protein and lipid content of whole brains of lean and $o b / o b$ mice. Left panel: total TCA-precipitable protein $(\mathrm{mg} / \mathrm{g}$ wet wt) from whole brain of lean (open bars, $\mathbf{n}=6$ ) and $o b / o b$ (hatched bars, $\mathrm{n}=11$ ) mice. Right panel: total methanol/ chloroform-extractable lipid content from lean (open bars, $\mathrm{n}=$ 12) and $o b / o b$ (hatched bars, $\mathrm{n}=11$ ) mice. $* \mathrm{P}<0.01$, lean vs $o b / o b$, t-test

cretion are accompanied by altered structural and/or functional neural correlates, may be possible.

The genetically obese (C57BL/6J ob/ob) mouse is chronically hyperinsulinaemic and demonstrates significant autonomic and endocrine abnormalities quite early in life [9]. Furthermore, the catecholamine content of the hypothalamus of $o b / o b$ mice has significant alterations relative to controls [25]. To investigate further the comparative neuroanatomy of this genetically hyperinsulinemic animal, we have used a computer-assisted morphometric technique to measure the soma cross-sectional areas of individual neurones from several CNS regions thought to influence feeding behaviour and insulin secretion [3].

The brain from 9-week old male $o b / o b$ mice (Jackson Laboratories, Bar Harbor, Maine) were removed and either paraffin embedded for morphometric analyses or used to measure brain weight, brain volume, and lipid and protein content. The total brain weight and volume from $o b / o b$ mice was significantly reduced compared to that of controls. One source of this reduced brain weight may be the reduced total lipid content (Figure 6). Interestingly, total protein content was unchanged, suggesting no overall reduction in the number of cellular elements. 
Morphometric analyses of cross-sectional areas of soma are summarized in Figure 7. As the soma crosssectional areas of cells from $o b / a b$ mice were significantly smaller for all sample regions except the lateral hypothalamic area (LHA), the results are shown as a percentage decrease. Among the six diencephalic regions sampled, the ventrolateral subdivision of the ventromedial hypothalamic nucleus (VMN-VL) had the greatest reduction in cell soma cross-sectional area relative to lean controls. The cell size distribution from LHA neurones was not changed in $o b / o b$ brains relative to controls, while neurones sampled from all surrounding cellular groups had reduced soma cross-sectional areas. Thus, one could consider that, relative to its own brain, the $o b / o b$ mouse has enlarged LHA neurones. Cells from the caudal DMX (a vagal brain stem nucleus) in $o b / o b$ mice showed the next smallest change (after the LHA), but still had significantly reduced soma cross-sectional areas compared to DMX neurones from control animals.

In spite of the dramatic reduction in soma crosssectional area of VMN neurones of $o b / o b$ mice, results from a current Golgi study suggest than VMN neurones have an equivalent number of dendrites compared to controls, but both LHA and VMN neurones demonstrate subtle orientation differences between $o b / o b$ and controls [4].

These anatomical findings demonstrate that at least in the adult $o b / o b$ mouse, there exist significant structural alterations in the CNS that may be related to the severe endocrinological disturbances seen in this genetically obese animal.

\section{Conclusions}

We have presented data using several methods of approach showing that the CNS can profoundly influence the output of pancreatic hormones, and furthermore, that it apparently does so via several output pathways. To summarize briefly: a) both brain stem (Amb) and LHA electrical stimulation cause increased insulin levels. A sympathetic inhibitory response accompanies such stimulation as shown by the further enhancement of insulin output in the presence of an $\alpha$-adrenergic blocker. b) The parallel between the effects of Amb and LHA stimulation is not complete since Amb-induced release of insulin is blocked by vagotomy while the LHA-induced response is not and may be humorally mediated; c) Acute VMH lesions as well as chronic VMH lesions promote an increase in insulin levels that can be blocked by vagotomy. These findings suggest that one primary consequence of $\mathrm{VMH}$ lesions is an

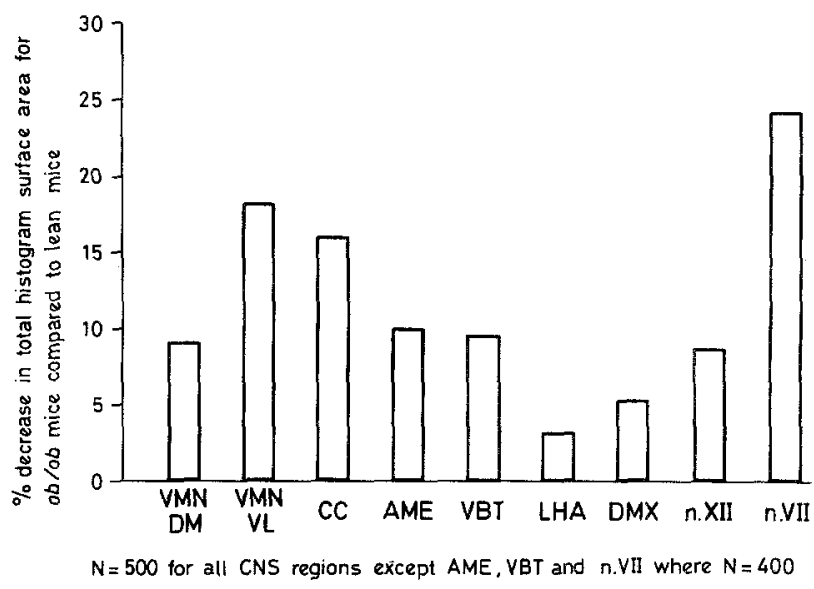

Fig. 7. Percentage decrease in total histogram surface area for $o b /$ $o b$ mice compared to lean mice in each of the nine CNS regions sampled for morphometric analysis. $\mathrm{n}=500$ for all regions except $\mathrm{AME}, \mathrm{VBT}$ and $\mathrm{n}$.VII, where $\mathrm{n}=400$. Abbreviations: $\mathrm{AME}=$ medial amygdaloid nucleus; $\mathrm{CC}=$ cingulate cortex; $\mathrm{DMX}=$ dorsal motor nucleus of the vagus nerve; LHA = lateral hypothalamic area; $\mathrm{n}$. VII $=$ motor nucleus of the facial nerve; $\mathbf{n}$. XII $=$ motor nucleus of the hypoglossal nerve; VBT = ventrobasal thalamus; $\mathrm{VMN}-\mathrm{DM}=$ dorsomedial subdivision of the ventromedial hypothalamic nucleus; VMN-VL $=$ ventrolateral subdivision of the ventromedial hypothalamic nucleus

increase in vagal activity, as expressed by increased insulin release. However, such increased vagal tone may be thought to have at least two components: 1) a rapid onset, phasic expression, by increasing the electrical activity to target organs in the abdomen, facilitatory for insulin release, as seen in the acute VMH lesion paradigm, and 2) a longer latency consequence of this increased vagal tone that would promote a hypertrophy of target organs, possibly via the release of some trophic factor, as is seen in the chronic VMH lesion paradigm.

Acknowledgements. The authors wish to thank Mrs Maria Becker, Miss Dominique Rutschman and Mrs Christine Barras for their excellent technical assistance. This work has been supported by grants 3.154.0.77 and 3.951.0.80 of the Swiss National Science Foundation, Berne (Switzerland), grant 1 R01 AM 25220-01, 02 of the National Institute of Arthritis, Metabolic and Digestive Diseases, Bethesda, MD (USA), and by a grant-in-aid of Nestlé S. A., Vevey (Switzerland).

\section{References}

1. Anand BK, Brobeck JR (1951) Hypothalamic control of food intake in rats and cats. Yale J Biol Med 24: 123-140

2. Beckstead RM, Norgren R (1979) An autoradiographic examination of the central distribution of the trigeminal, facial, glossopharyngeal and vagus nerves in the monkey. $J$ Comp Neurol 184: 455-472

3. Bereiter DA, Jeanrenaud B (1979) Altered neuroanatomical organization in the central nervous system of the genetically obese $(o b / o b)$ mouse. Brain Res 165: 249-260 
4. Bereiter DA, Jeanrenaud B (1980) Altered dendritic orientation of hypothalamic neurons from genetically obese $(a b / o b)$ mice. Brain Res 202: 201-206

5. Bergman RN, Miller RE (1973) Direct enhancement of insulin secretion by vagal stimulation of the isolated pancreas. Am J Physiol 225: 481-486

6. Berthoud H-R, Bereiter DA, Jeanrenaud B (1980) Role of the autonomic nervous system in the mediation of LHA electrical stimulation - induced effects on insulinemia and glycemia. J Autonomic Nerv Sys 2: 183-198

7. Berthoud H-R, Bereiter DA, Trimble ER, Siegel EG, Jeanrenaud B (1981) Cephalic phase, reflex insulin secretion: Neuroanatomical and physiological characterization. Diabetologia 20 [Suppl]: 393-401

8. Berthoud H-R, Jeanrenaud B (1979) Acute hyperinsulinemia and its reversal by vagotomy after lesions of the ventromedial hypothalamus in anesthetized rats. Endocrinology 105: 146-151

9. Bray GA, York DA (1979) Hypothalamic and genetic obesity in experimental animals: an autonomic and endocrine hypothesis. Physiol Rev 59: 719-809

10. Coil JD, Norgren R (1979) Cells of origin of motor axons in the subdiaphragmatic vagus of the rat. J Autonomic Nerv Sys 1: $203-210$

11. Frohman LA, Ezdinli JR (1967) Effect of vagotomy and vagal stimulation on insulin release. Diabetes 16: 443-448

12. Gold RM (1973) Hypothalamic obesity: the myth of the ventromedial nucleus. Science 182: 488-490

13. Gold RM, Jones AP, Sawchenko PE, Kapatos G (1977) Paraventricular area: Critical focus of a longitudinal neurocircuitry mediating food intake. Physiol Behav 18: 1111-1119

14. Han PW, Yu Y-K, Chow SL (1970) Enlarged pancreatic islets of tube-fed hypophysectomized rats bearing hypothalamic lesions. Am J Physiol 218: 769-771

15. Hill DE, Mayes S, Di Battista D, Lockhart-Ewart R, Martin JM (1977) Hypothalamic regulation of insulin release in rhesus monkeys. Diabetes 26: 726-731

16. Idahl LA, Martin JM (1971) Stimulation of insulin release by a ventrolateral hypothalamic factor. J Endocrinol 51: 601-602

17. Inoue S, Bray GA (1977) The effects of subdiaphragmatic vagotomy in rats with ventromedial hypothalamic obesity. Endocrinology 100: 108-114

18. Inoue S, Bray GA, Mullen YS (1978) Transplantation of pancreatic $\beta$-cells prevents development of hypothalamic obesity in rats. Am J Physiol 235: E266-E271

19. Iversen J (1973) Adrenergic receptors and the secretion of insulin and glucagon from the isolated, perfused canine pancreas. J Clin Invest 52: 2102-2116

20. Iversen $\mathbf{J}$ (1973) Effect of acetylocholine on the secretion of glucagon and insulin from the isolated, perfused canine pancreas. Diabetes 22: 381-387

21. Järhult J, Holst JJ (1978) Reflex adrenergic control of endocrine pancreas evoked by unloading of carotid baroreceptors in cats. Acta Physiol Scand 104: 188-202

22. Kaneto A, Kosaka K, Nakao K (1967) Effects of stimulation of the vagus nerve on insulin secretion. Endocrinology 80: $530-536$

23. Laughton W, Powley TL (1979) Four central nervous system sites project to the pancreas. Soc Neurosci Abstr 5: 46

24. Loewy AD, Burton $H$ (1978) Nuclei of the solitary tract: efferent projections to the lower brain stem and spinal cord of the cat. J Comp Neurol 181: 421-449

25. Lorden JF, Oitmans GA, Margules DL (1975) Central catecholamine levels in genetically obese mice $(o b / o b$ and $d b /$ db). Brain Res 96: 390-394

26. Louis-Sylvestre J (1976) Preabsorptive insulin release and hypoglycemia in rats. Am J Physiol 230: 56-60

27. Miller RE, Waid TH, Joyce MP (1976) Direct neural inhibition of insulin secretion in response to systemic hypoglycemia. Am J Physiol 230: 1090-1094

28. Powley TL (1977) The ventromedial hypothalamic syndrome, satiety, and a cephalic phase hypothesis. Psychol Rev 84: 89-126

29. Rohner-Jeanrenaud F, Jeanrenaud B (1980) Consequences of ventromedial hypothalamic lesions upon insulin and glucagon secretion by subsequently isolated perfused pancreases in the rat. J Clin Invest 65: 902-910

30. Saper CB (1979) Anatomical substrates for the hypothalamic control of the autonomic nervous system. In: McBrooks C, Koizumi K, Sato A (eds) Integrative functions of the autonomic nervous system. Univ of Tokyo Press, Tokyo, p 333-341

31. Swanson LW (1977) Immunohistochemical evidence for a neurophysin-containing autonomic pathway arising in the paraventricular nucleus of the hypothalamus. Brain Res 128: 346-353

32. Ungerstedt U (1971) Stereotaxic mapping of the monoamine pathways in the rat brain. Acta Physiol Scand [Suppl] 367: $1-48$

33. Woods SC, Porte D Jr (1974) Neural control of the endocrine pancreas. Physiol Rev 54: 596-619

Prof. B. Jeanrenaud

Laboratoires de Recherches Métaboliques

Avenue de la Roseraie 64

$\mathrm{CH}-1205$ Geneva

Switzerland

\section{Discussion after Both of the Presentations by the Jeanrenauds}

Shimazu: In rabbits, electrical stimulation of the LHA caused no change of insulin or glucagon secretion. The stimulation parameters were $50 \mathrm{~Hz}, 0.1 \mathrm{msec}$ and $0.2 \mathrm{~mA}$. Comparable stimulation in the $\mathrm{VMH}$ caused a rapid increase of glucagon and glucose levels and a delayed increase of insulin which didn't begin until the stimulation had ceased. The latter was probably secondary to the elevated glucose levels.

B. Jeanrenaud: Your data are very comparable to ours except that the peak insulin and glucagon levels we observed are somewhat higher. On the other hand, we used an alpha adrenergic blocker and you did not, so that probably accounts for the difference.

Shimazu: Another important difference is that our animals were unanaesthetized and free-moving whereas yours were anaesthetized.

Steffens: In our experiments in which we used norepinephrine to stimulate the hypothalamus, we do not see the sympathetic component which you have observed. Rather, we have found only parasympathetic effects. 
B. Jeanrenaud: Yes, the difference is probably due to your use of a chemical stimulant with presumably activation of only a subset of the neurons we activate electrically; alternatively, as Dr. Shimazu pointed out, your use of unanaesthetized animals may make a big difference. But I expect that we are simply stimulating both sympathetic and parasympathetic areas.

Berthoud: Years ago, Booth and Miller stimulated electrically the LHA of freely moving rats and observed a very large increase of plasma glucose (Physiol Behav (1969) 4: 991), suggesting that a sympathetic response can be seen in unanesthetized rats, so anaesthesia is probably not the explanation.

Niijima: We have done some experiments which may relate to these differences. We recorded from the hepatic branch of the splanchnic nerve, which is presumably only sympathetic in nature. When we added glucose to the carotid artery, there was decreased activity in the nerve and when we added 2-deoxyglucose, there was increased activity. Therefore, glucose availability to the brain could influence sympathetic outflow. When we made the same manipulations and recorded from the vagus, there was no remarkable change of activity, and presumably, therefore, no change of parasympathetic activity. So it would seem that the nature of the stimulus to the brain might be expected to activate selective efferent pathways.

Nicolaidis: In your experiment you suggest that perhaps a hypothalamic or a pituitary factor might be influencing the secretion of insulin from the pancreas. What do you know about that factor?

Berthoud: As you know, there is evidence in the literature for both hypothalamic (J Endocrinol (1971) 51: 601) and pituitary (Horm Metab Res (1976) 8: 329) factors. There is even a suggestion in the genetically obese mouse that one factor has been identified. Beloff-Chain says that CLIP or corticotropin-like immunoreactive peptide from the pituitary may be important in increasing insulin secretion (In: Festing MW (ed) (1979) Animal models of obesity. Mc Millan, London, p 91).

B. Jeanrenaud: Yes, CLIP or any other natural peptide or fragment which can be isolated from the pituitary or hypothalamus is a potential candidate.

Porte: I think there's a potential for misinterpreting these experiments. When you administer both an alpha blocker plus glucose, the B-cells become very activated and very sensitive. Further, catecholamines in the blood are very elevated and only the beta-adrenergic effects can be seen. Under these conditions, any factor which has the ability to stimulate adenyl-cyclase might look like a specific B-cell stimulant, but may not have any physiological significance normally.

B. Jeanrenaud: This is somewhat of an overinterpretation as you disregard the fact that, under alphablockade, the stimulatory component of L.H stimulation on insulin release remained, even after chordotomy or superimposed betablockade.
Porte: I would like to ask your explanation for the increased sensitivity of the islets in vitro taken from rats with VMH lesions.

F. Jeanrenaud: We think that there is probably an altered number of muscarin receptors. To test this, we gave a large infusion of methacholine and observed a very large increase of both insulin and glucagon secretion, both of which were blocked with atropine. There was a little decrease of somatostatin levels with this treatment and atropine did also reverse this effect. We interpret these findings to mean that $\mathrm{A}$ and $\mathrm{B}$-cells have increased numbers of receptors and that the D-cells are normally inhibited by acetylcholine.

Samols: In at least one of the earlier papers, very low levels of calcium were used in the perfusion fluid and it was very unphysiological. In fact, they also reported no effect of acetylcholine on insulin or glucagon. On the other hand, you use extremely large amounts of methacholine and may be providing an unphysiological stimulus in the other direction.

Porte: Yes, with so much methacholine, you might well be releasing other neurotransmitters from intrinsic nerves in your tissue and getting diverse effects; and these might not necessarily be effected by atropine.

F. Jeanrenaud: That is a possibility.

Goldman: Have you looked at the morphology of these tissues to see if any hyperplasia exists?

F. Jeanrenaud: We are doing this now with Dr. D. Coleman. We have found what appears to be an increase of Acell content of the islets. We also have evidence that the number of D-cells might be increased one week after the lesion.

Smith: A group of us including Steve Woods, Yutaka Seino, Dave West and John Ensinck in Seattle have been looking at the somatostatin content of the islets in VMHlesioned rats. We have found an inverse relationship between pancreatic content of somatostatin-like immunoreactivity (SSLI) and the degree of adiposity, and that this relationship is independent of VMH lesions. Rats with lesions that were fed to be as heavy as controls had normal levels of pancreatic SSLI. Obese VMH-lesioned rats had slightly reduced levels; and starved unlesioned rats had greatly elevated SSLI levels (West et al. Diabetes (1979) 28: 373). Dr. Seino has data on D-cell number which parallel these in the VMH rat, and we are finding the same thing with a different morphometric method. So our findings appear to conflict somewhat with yours. One difference may be the time after the lesioning at which the tissues were examined. We waited until 6 weeks post-lesion to sacrifice the animals.

F. Jeanrenaud: Yes, at least at one week after the lesion, our lesioned rats had slightly elevated total pancreatic somatostatin content.

Woods: More importantly, as I recall, your rats were underfed to control levels. If that's true, your data actually fit well with ours.

F. Jeanrenaud: That is true. 